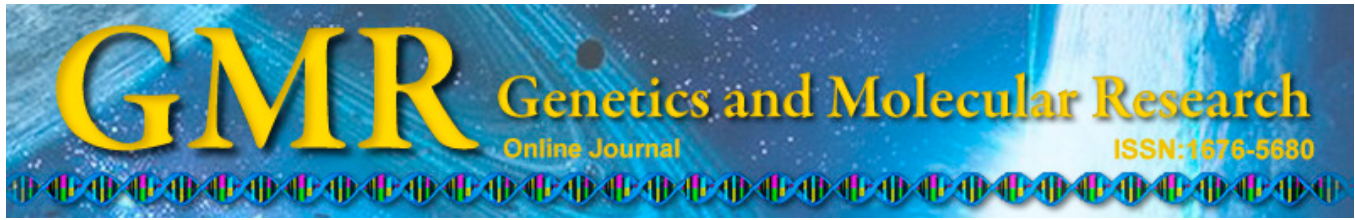

\title{
DKK1 eukaryotic expression plasmid and expression product identification
}

\author{
G.Y. Bao, K.Y. Lu, S.F. Cui and L. Xu \\ Department of Clinical Laboratory, Yangzhou First People's Hospital, \\ Yangzhou, Jiangsu, China \\ Corresponding author: G.Y. Bao \\ E-mail: baogy_fml@163.com
}

Genet. Mol. Res. 14 (2): 6312-6318 (2015)

Received April 7, 2014

Accepted October 8, 2014

Published June 11, 2015

DOI http://dx.doi.org/10.4238/2015.June.11.5

\begin{abstract}
We constructed the human dickkopf 1 (DKK1) eukaryotic expression plasmid and expressed, purified, and identified its expression product. We extracted cancer cells from cervical cancer tissue, followed by extraction of mRNA. Reverse transcription-polymerase chain reaction was conducted to obtain DKK1 gene fragments. Using these fragments, we prepared the recombinant plasmid pCMV-HA2/ DKK1. The recombinant plasmid was restriction enzyme-digested and sequenced, and using liposome vectors, was transiently transfected into Free-Style 293-F cells (serum-free medium). DKK1 protein was detected by western blotting. The amplification product showed the expected size. Restriction enzyme digestion and sequence analysis showed that the recombinant plasmid was PCMV-HA2/DKK1. The expression product was verified properly by western blotting using an anti-DKKI antibody. The successful cloning of the DKKI gene and expression of DKKI protein will be useful for studying the biological activity of tumorigenesis.
\end{abstract}

Key words: Cells; Culture Technology; Dickkopf-1 protein; Eukaryotic expression; Mammal cells 


\section{INTRODUCTION}

Dickkopf-1 (DKK1) is a secretory glycoprotein that is a member of the DKK family. It contains 2 conserved regions, including Cys 1 and Cys2, which are rich in cysteine, and can inhibit the classical Wnt signaling pathways (Fedi et al., 1999; Niehrs, 2006). Previous studies have shown that DKK1 is a tumor-specific protein that cannot be expressed in normal tissues of adults other than the embryo; in adults, this protein is highly expressed in liver cancer, lung cancer, breast cancer, ovarian cancer, cervical cancer, stomach cancer, glioma, melanoma, osteosarcoma, and bladder cancer, among others, and also is associated with the metastasis and prognosis of hepatocellular carcinoma (Yu et al., 2006, 2009; Sheng et al., 2009; Xu, 2011). Obtaining human DKK1 protein in its functional biological form will enable studies of DKK1 structure and function, as well as its role in tumors. The protein can also be used to prepare antibodies to facilitate early diagnosis and specific targeted therapy for tumors highly expressing DKK1. Because the DKK1 protein is rich in disulfide bonds, the folding process after translation is complex and shows glycosylation modification. Therefore, it is difficult to obtain the active protein in the natural state using non-mammalian cell expression systems. Thus, our goal was to construct an eukaryotic expression system for DKK1 using the mammalian cells Free-Style 293-F to express the human DKK1 protein. Our results will enable efficient preparation of recombinant DKK1 and further study of this protein in tumor biology.

\section{MATERIAL AND METHODS}

Cervical cancer tissue was confirmed to be cervical squamous cell carcinoma tissue by pathological diagnosis. Dulbecco's Modified Eagle Medium, fetal bovine serum, and TRIzol for RNA extraction were obtained from Gibco BRL (Grand Island, NY, USA), Lipofectamine 2000 was obtained from Invitrogen (Carlsbad, CA, USA), HA antibodies were from Santa Cruz (Santa Cruz, CA, USA), and the anti-mouse secondary antibody was from Pierce (Rockford, IL, USA). The plasmid extraction kit was produced by Shanghai HuaShun Corporation (Shanghai, China), the DGL2000 Marker was from Beijing Dingguo Company (Beijing, China), Top10F cells were purchased from Clontech (La Jolla, CA, USA), LB culture medium was from Decent Biotech (Beijing, China), and pCMV-HA2 was bought from Biyuntian Biotechnology Research Institute (Shanghai, China). Free-Style 293-F cells, transfection reagent 293-fectin, and culture medium Free-Style 293 Expression Medium were purchased from Invitrogen; plasmid extraction and gel extraction kits, the reverse transcription-polymerase chain reaction (RT-PCR) kit, T4 DNA ligase, and the restriction enzyme EcoRI were from TaKaRa (Shiga, Japan). Primer synthesis and gene sequencing were carried out by Shanghai Boya (Shanghai, China).

\section{Construction of eukaryotic expression plasmid}

\section{Preparation of cervical cancer tissue cells}

We cut the cervical cancer tissue into pieces and subjected the sample to enzyme digestion with $0.05 \%$ type I collagenase. This solution was filtered through a 200 -mesh filter screen and the cellular constituents were inoculated into Dulbecco's Modified Eagle Medium containing $10 \%$ fetal bovine serum. The cells were cultured in an incubator with $5 \% \mathrm{CO}_{2}$ at $37^{\circ} \mathrm{C}$; the liquid was replaced every 3 days and the 5th-generation cells were collected. 


\section{Generation of DKK1 by RT-PCR}

From $1 \times 10^{7}$ cells, mRNA was extracted using a conventional method; the nutrient solution was discarded on a 35-mm Petri dish. The sample was washed twice with phosphatebuffered saline (PBS) and $0.5 \mathrm{~mL}$ TRIzol was added directly to the cells on the Petri dish. The solution was transferred to a $1.5-\mathrm{mL}$ centrifuge tube for mixing. The cells were incubated at room temperature for $5 \mathrm{~min}$ and then $100 \mu \mathrm{L}$ chloroform was added. This solution was mixed by inverting the centrifuge tube; $5 \mathrm{~min}$ later, the tubes were centrifuged at 13,000 $\mathrm{g}$ for $10 \mathrm{~min}$. The upper aqueous phase was carefully removed to another $1.5-\mathrm{mL}$ centrifuge tube and $250 \mu \mathrm{L}$ isopropyl alcohol was added. The tube was mixed and then incubated at room temperature for $5 \mathrm{~min}$, followed by high-speed centrifugation for $5 \mathrm{~min}$. The supernatant was discarded. To the pellet, $1 \mathrm{~mL} \mathrm{75 \%}$ ethanol was added, mixed, centrifuged at $7500 \mathrm{rpm}$ for $5 \mathrm{~min}$. The supernatant was again discarded and the sample was incubated at room temperature for 5-15 min until RNA precipitate was dry. Diethylpyrocarbonate-treated water was added to dissolve the RNA and the samples were stored at $-70^{\circ} \mathrm{C}$. The RT-PCR kit was used to prepare cDNA. The following primers were used for RT- PCR: forward, 5'-TCACGCTATGTGCTGCCCCG-3'; reverse, 5'-TGAGGCACAGTCTGATGACCGGA-3'. Next, $8 \mu \mathrm{L} 2 \mu \mathrm{g}$ RNA was mixed with DNase I, $1 \mu \mathrm{L}$ oligo dT, and $10 \mathrm{mM}$ dNTP Mix $1 \mu \mathrm{L}$, heated at $65^{\circ} \mathrm{C}$ for $5 \mathrm{~min}$, and then immediately placed on ice for $10 \mathrm{~min}$. After this, $10 \mu \mathrm{L}$ reverse transcription mixture $(2 \mu \mathrm{L} 10 \mathrm{X}$ RT buffer, 4 $\mu \mathrm{L} 25 \mathrm{mM} \mathrm{MgCl}, 2 \mu \mathrm{L} 0.1 \mathrm{M}$ DTT, $1 \mu \mathrm{L} 40 \mathrm{U} / 2 \mu \mathrm{L}$ RNase OUT, and $1 \mu \mathrm{L} 200 \mathrm{U}$ Super-Script reverse transcription III) were mixed, heated at $50^{\circ} \mathrm{C}$ for $50 \mathrm{~min}$, and reacted for another $5 \mathrm{~min}$ at $85^{\circ} \mathrm{C}$. The samples were immediately placed on ice for $10 \mathrm{~min}$. Next, $1 \mu \mathrm{L}$ RNase $\mathrm{H}$ was added to remove the RNA for $20 \mathrm{~min}$ at $37^{\circ} \mathrm{C}$.

\section{Obtaining targeted DKK1 fragment}

Primers were designed for the cDNA template for PCR amplification that covered the entire open reading frame of DKK1: forward primers: 5'-GAATTCATGGCTCTGGGCGCA-3' reverse primer: 5'-GAATTCGTGTCTCTGACAAGT-3', according to the requirements of eukaryotic expression vectors pCMV-HA2. Primers were designed for PCR amplification of DKK1. An EcoRI site was included at the 5 ' and 3 ' ends. The PCR system included $0.5 \mu \mathrm{LcDNA}$ template, $1 \mu \mathrm{L} 10 \mathrm{mM}$ forward and reverse primers, $1 \mu \mathrm{L} 10 \mathrm{mM}$ dNTP, $2.5 \mu \mathrm{L} 10 \mathrm{X}$ buffer, and $0.5 \mu \mathrm{L}$ high-fidelity enzyme; deionized water was added for a final volume of $25 \mu \mathrm{L}$. The PCR conditions were as follows: $94^{\circ} \mathrm{C}$ for $5 \mathrm{~min} ; 94^{\circ} \mathrm{C}$ for $3 \mathrm{~s}, 55^{\circ} \mathrm{C}$ for $30 \mathrm{~s}, 68^{\circ} \mathrm{C}$ for $1 \mathrm{~min}$, 35 cycles; $68^{\circ} \mathrm{C}$ for $7 \mathrm{~min}$. PCR products were separated by $1 \%$ agarose gel electrophoresis in $1 \mathrm{X}$ TAE electrophoretic buffer solution. Bands were observed under ultraviolet light, extracted from the gel, and placed in collection tubes. The specific steps and operation of gel extraction were conducted according to the manufacturer instruction. After PCR amplification, and digestion of PCR products, concentration of the products was determined.

\section{Construction of pCMV-HA2/DKK1 recombinant plasmid}

Next, $3.5 \mu \mathrm{L}$ purified PCR product was mixed with $0.5 \mu \mathrm{L}$ pCMV-HA2 carrier, $0.5 \mu \mathrm{L}$ $\mathrm{T} 4$ ligase, and $0.5 \mu \mathrm{L} 10 \mathrm{X}$ reaction buffer. The sample was mixed until it was concentrated on the bottom of the tube, and then incubated overnight at $16^{\circ} \mathrm{C}$. The next day, the ligated products were transformed into Top $10 \mathrm{~F}$ competent cells with shaking for $1 \mathrm{~h}$ at $37^{\circ} \mathrm{C}$. The cells 
were plated onto an LB plate containing ampicillin and cultured overnight at $37^{\circ} \mathrm{C}$. White bacterial colonies were added to $3 \mathrm{~mL}$ LB culture medium containing ampicillin, cultured overnight with shaking at $37^{\circ} \mathrm{C}$, and the plasmid was extracted using an extraction kit according to manufacturer instructions. After identification of enzyme digestion products, the samples were sequenced by Shanghai Boya Company.

\section{Expression and identification of DKK1 protein}

For transfection of the recombinant vector pCMV-HA2/DKK1, Free-Style 293-F cells were incubated at $37^{\circ} \mathrm{C}$ and $\mathrm{CO}_{2}$ volume fraction of $8 \%$. The flasks were shaken at $130 \mathrm{rpm}$. Before transfection, cell density was adjusted to $1 \times 10^{6} / \mathrm{mL}$. Next, $100 \mathrm{pg}$ recombinant plasmid pCMV-HA2/DKK1 and $200 \mu$ L Free-Style 293-F cells for liposome transfection reagent were diluted to $3.33 \mathrm{~mL}$ with Opti-MEM, allowed to stand for $5 \mathrm{~min}$, and the plasmid and transfection reagents were mixed slowly and reacted for $20 \mathrm{~min}$ at room temperature. Next, $93.3 \mathrm{~mL}$ Free-Style $293-\mathrm{F}$ cells (density of $1 \times 10^{6} / \mathrm{mL}$ ) was added to the DNAfectin reagent mixture to a volume of $100 \mathrm{~mL}$. The cells were cultured at $130 \mathrm{rpm}$ at $37^{\circ} \mathrm{C}$ and with $8 \% \mathrm{CO}_{2}$.

After DKK1 protein expression, transfected cells were lysed with lysis buffer and then centrifuged. The supernatant was used to determine protein concentration using the BCA protein quantitative method. The cell culture supernatant fluid or cell protein extraction was mixed with loading buffer $(0.35 \mathrm{M}$ Tris- $\mathrm{HCl}, \mathrm{pH} 6.8,10.28 \%$ sodium dodecyl sulfate, $36 \%$ glycerol, $0.6 \mathrm{M}$ dithiothreitol, and $0.012 \%$ bromophenol blue), boiled for $5 \mathrm{~min}$ at $100^{\circ} \mathrm{C}$, and then separated by $12 \%$ sodium dodecyl sulfate-polyacrylamide gel electrophoresis (stacking gel, $80 \mathrm{~V}, 25 \mathrm{~min}$; separating gel, $150 \mathrm{~V}, 1 \mathrm{~h}$ ), and then the protein was electrotransferred to a nitrocellulose membrane at $300 \mathrm{~mA}$ for $1 \mathrm{~h}$. The membrane was blocked with PBS-5\% Tween-20 containing 5\% skim milk powder for 2-3 h. Rabbit anti-human DKK1 PcAb (diluted 1:1000) was added and incubated overnight at $4^{\circ} \mathrm{C}$. The next day, the membrane was washed with PBS-5\% Tween-20 4 times, horseradish peroxidase-conjugated goat anti-rabbit IgG (diluted 1: 2000) was added, and the sample was incubated at room temperature for $1 \mathrm{~h}$. The membrane was washed with PBS-5\% Tween-20 5 times and developed according to the manufacturer protocol.

\section{RESULTS}

\section{RT-PCR results}

The RT-PCR results revealed is a specific band close to 250 base pairs in all amplification products of tumor cells following reverse transcription of DKK1 cDNA. These bands were observed on $1.5 \%$ agarose gel electrophoresis.

\section{Identification of pCMV-HA2 carrier expression clone enzyme}

PCR of DKK1 showed a single band on the gel. PCR products were purified and ligated into pCMV-HA2. After transformation, colonies were picked and subjected to enzymatic digestion. Agarose gel electrophoresis revealed a band at 756 base pairs, which was the expected size (Figure 1). 


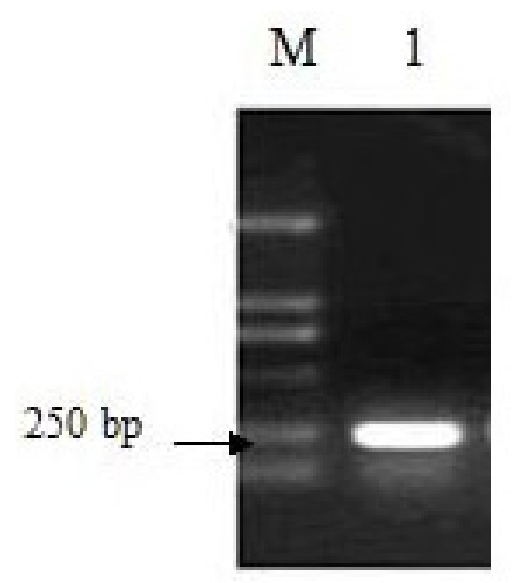

Figure 1. RT-PCR detection of tumor cells DKK1 gene expression. Lane $M=$ DG12000; lane 1 = cervical carcinoma cell RT-PCR results.

The sequences of the products were found to be identical to the human DKK1 cDNA sequence (NM_012242. 2) obtained from the GenBank database, verifying that the construction of the $\mathrm{pCM} V$ - HA2/DKK1 recombinant plasmid was successful.

Human DKK1 protein was secreted from Free-Style 293-F cells. To verify that the constructed eukaryotic vector expressed and secreted DKK1 protein, the recombinant plasmid pCMV-HA2/DKK1 and an empty pCMV-HA2 as a control were transfected into Free-Style 293-F cells for $48 \mathrm{~h}$. The supernatant and cells were harvested, subjected to protein detection by Far-western blotting. DKK1 protein expression was detected in both the supernatant and the cell cytoplasm (Figure 2). The molecular weight of the products was approximately 28 $\mathrm{kDa}$, which is consistent with the expected value. Additionally, the DKK1-HA fusion protein was detected and verified using an HA antibody (Figure 3).

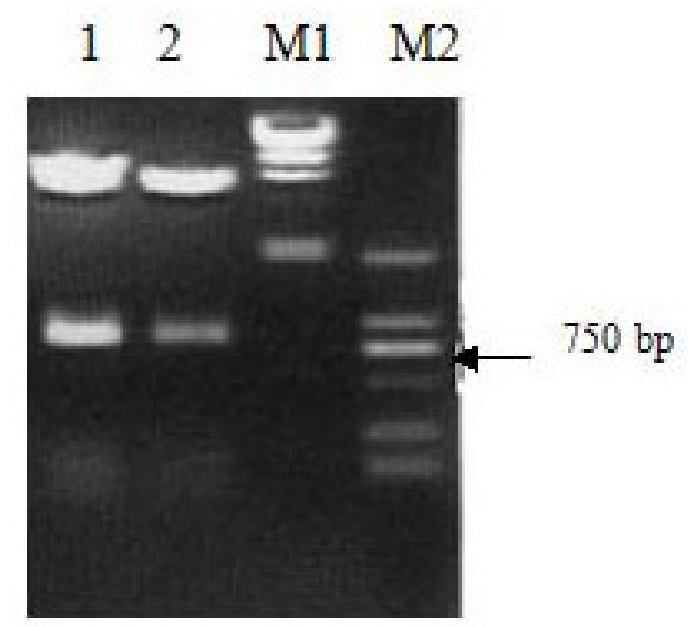

Figure 2. pCMV-HA2 / Dkk1 subcloned 1,2 digested by EcoRI electrophoresis. Lane M1 = $\mathrm{DNNA}$; lane M2= DL2000; lanes 1 and 2 = pCMV-HA2/DKK1 two expression clones. 


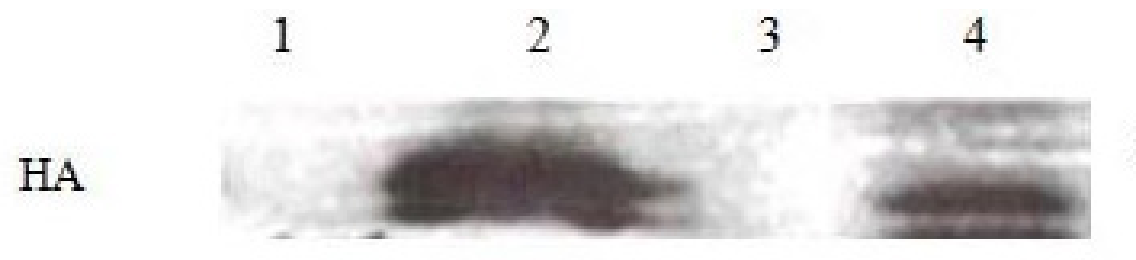

\section{$28 \mathrm{kDa}$}

\section{DKK1}

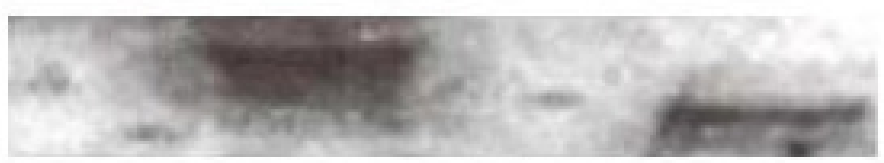

\section{$28 \mathrm{kDa}$}

Figure 3. Western blot detection of HA-tagged fusion proteins DKK1. Lanes 2 and $4=$ pCMV-HA2/DKK1 expression clones; lanes 1 and $3=$ control.

\section{DISCUSSION}

The Wnt signaling pathway plays a very important role in the individual development and occurrence of a wide variety of tumors. The Wnt pathway involves 2 extracellular inhibitors (Kawano and Kypta, 2003), including frizzled-related proteins and DKK1. The molecular weight of DKK 1 is approximately $35 \mathrm{kDa}$ and is a secretory glycoprotein. It combines with Wnt receptor LRP5 (or LRP6) on the cell membrane and DKK1 co-receptor Kremen1 (or Kremen2) to form an endocytosis globule, blocking the Wnt signaling pathway. Previous studies have shown that DKK1 is only expressed in the placenta tissues in adults, but abnormally expressed in various tumors. The introduction of a plasmid that lacking expression in HeLa cells cultured in vitro can lead to abnormal proliferation of tumor cells (Mikheev et al., 2004), and silent expression of DKK1 has been observed in cervical cancer HeLa cell lines (Lee et al., 2009). In isolated non-tumor revertants found in cultures of cervical cancer HeLa cell lines, high expression of DKK1 protein has been observed (Mikheer et al., 2004), indicating an important role of DKK1 in the occurrence of cervical cancer. Various studies have shown that the DKK1 protein is likely to be tumor-specific and can be used for the diagnosis of tumors or targeted therapy. Therefore, obtaining human DKK1 protein in its natural, bioactive state is critical for studying its biological structure and function as well as its application in tumor recognition.

A variety of expression systems is available for protein preparation, including Escherichia coli, yeast, and expression systems in plant, insect, and mammalian cells. Among these, the mammalian cell expression system is more closely related to natural active protein in terms of protein solubility, protein folding, and modification after translation; thus, it has become increasingly widely applied (Fedi et al., 1999). HEK293 cells and their derived cells are common mammalian cells used for protein preparation (Wurm, 2004).

In this study, we used the eukaryotic expression vector pCMV-HA2 and mammalian cell Free-Style 293-F expression system to obtain large amounts of human DKK1 recombinant protein that was similar to the natural active protein. Cancer cells were extracted from cervical cancer tissues, and total mRNA was extracted using a conventional method. Human DKK1 gene fragments were obtained by RT-PCR and PCR. Next, we constructed the recombinant plasmid pCMV-HA2/DKK1 and extracted the recombinant plasmid after bacteria amplification. After enzyme digestion and electrophoresis, the fragment was found to be approximately 750 base pairs, which was the expected length. The sequencing result was com- 
pletely consistent with the human DKK1 gene cDNA sequence (NM_012242.2), indicating that pCMV-HA2/DKK1 recombinant plasmid construction was successful. Transient transfection of vectors into Free-Style 293-F cells (serum-free culture) with liposomes was conducted and DKK1 protein was detected using Far-western blotting of the supernatant and cell lysate after culture. The expression product after identification of western blotting was the DKK1 protein. We successfully constructed the DKK1 eukaryotic expression plasmid and identified the eukaryotic expression product of the gene, enabling the future study of DKK1 protein biology activity in tumors.

\section{REFERENCES}

Fedi P, Bafico A, Nieto Soria A, Burgess WH, et al. (1999). Isolation and biochemical characterization of the human Dkk1 homologue, a novel inhibitor of mammalian Wnt signaling. J. Biol. Chem. 274: 19465-19472.

Kawano Y and Kypta R (2003). Secreted antagonists of the Wnt signalling pathway. J. Cell Sci. 116: 2627-2634.

Lee J, Yoon YS and Chung JH (2009). Epigenetic silencing of the WNT antagonist DICKKOPF-1 in cervical cancer cell lines. Int. J. Cancer 124: 287-297.

Mikheev AM, Mikheeva SA, Liu B, Cohen P, et al. (2004). A functional genomics approach for the identification of putative tumor suppressor genes: Dickkopf-1 as suppressor of HeLa cell transformation. Carcinogenesis 25: 47-59.

Niehrs C (2006). Function and biological roles of the Dickkopf family of Wnt modulators. Oncogene 25: 7469-7481.

Sheng SL, Huang G, Yu B and Qin WX (2009). Clinical significance and prognostic value of serum Dickkopf-1 concentrations in patients with lung cancer. Clin. Chem. 55: 1656-1664.

Wurm FM (2004). Production of recombinant protein therapeutics in cultivated mammalian cells. Nat. Biotechnol. 22: 1393-1398.

Xu YQ (2011). Serum Dickkopf 1 level application in the diagnosis of lung cancer and bone metastases. Guo Ji JianYan Yi Xue 32: 379-380.

Yu B, Yang X, Xu Y, et a1. (2009). Elevated expression of DKK1 is associated with cytoplasmic/nuclear beta-catenin accumulation and poor prognosis in hepatocellular carcinomas. J. Hepatol. 50: 948-957.

Yu YJ, Wan SZ, Yu B, et al. (2006). Dickkopf (DKKI) in liver cancer tissues and a variety of expression analysis of tumor cells. Tumor 26: 1109-1112. 